\title{
Moderate sleep deprivation produces impairments in cognitive and motor performance equivalent to legally prescribed levels of alcohol intoxication
}

\author{
A M Williamson, Anne-Marie Feyer
}

\begin{abstract}
Objectives-To compare the relative effects on performance of sleep deprivation and alcohol.

Methods-Performance effects were studied in the same subjects over a period of 28 hours of sleep deprivation and after measured doses of alcohol up to about $0.1 \%$ blood alcohol concentration (BAC). There were 39 subjects, 30 employees from the transport industry and nine from the army.

Results-After 17-19 hours without sleep, corresponding to 2230 and 0100 , performance on some tests was equivalent or worse than that at a BAC of $0.05 \%$. Response speeds were up to $50 \%$ slower for some tests and accuracy measures were significantly poorer than at this level of alcohol. After longer periods without sleep, performance reached levels equivalent to the maximum alcohol dose given to subjects (BAC of $0.1 \%$ ).

Conclusions-These findings reinforce the evidence that the fatigue of sleep deprivation is an important factor likely to compromise performance of speed and accuracy of the kind needed for safety on the road and in other industrial settings.

(Occup Environ Med 2000;57:649-655)
\end{abstract}

Keywords: sleep deprivation; performance; alcohol

The implications of fatigue for safe performance are well recognised particularly in road safety, but in other settings as well. Fatigue is most likely to occur when rest is reduced such as when working long or irregular hours, doing shift and night work, or due to family responsibilities or lifestyle choices. Effects of fatigue are thought to play a part in between $16 \%$ and $60 \%$ of road accidents ${ }^{12}$ and in the United States were estimated to cost in the vicinity of $\$ 50$ billion. $^{3}$

Recently authors have argued that until now society has simply accepted the hazards of fatigue despite evidence of increased risk to health and safety. ${ }^{45}$ This has led to calls for better information on the extent and consequences of the effects of fatigue on performance. ${ }^{6}$ The problem, in practice, is at what level of fatigue does performance become a problem? In setting any safety standard for the fatigue caused by sleep deprivation, the sort of information needed is a comparison of performance after a known number of hours spent awake with that caused by some other agent that decreases performance.

Alcohol effects serve as a good model for an acceptable standard for safe performance. Alcohol effects have been measured and standardised by setting limits on alcohol consumption based on their predicted effects on driving performance. ${ }^{7}$ Many countries have set limits for alcohol levels while driving which are based on laboratory, simulator, and on road measures of speed and accuracy of performance. ${ }^{8}$ These standards provide a benchmark for performance deficits caused by injury, illness, or in this case, the fatigue of sleep deprivation. By comparing the change in performance due to alcohol consumption at concentrations widely agreed to be hazardous $(0.05 \%$ blood alcohol concentration (BAC) $)^{8}$ with the same behaviour after sleep deprivation, it should be possible to assess the amount of sleep deprivation at which equivalent deficits occur. This is the aim of our study. An earlier study ${ }^{9}$ used a similar study design but looked at effects on only a single test (eye-hand coordination). Single tests which are an amalgamation of functions and simple in terms of effort may not show sufficient information for setting standards in a range of work settings. The current study looked at effects on a range of performance tests including tasks involving cognitive and motor speed, accuracy, coordination, and attention.

\section{Method}

SUBJECTS

Thirty nine subjects participated in this study. Thirty seven were male and two were female. Table 1 shows demographic characteristics for the sample. Most subjects were in the 30-49 age group (59\%) and were living with a partner $(77 \%)$. Most subjects had only 10 years of education or less $(60 \%)$. Subjects were volunteers from a large road transport company (30 subjects) and the transport corps of the Australian army (nine subjects). In both cases, all subjects were volunteers from the drivers and administration staff available at the time. They were allowed paid work time to participate in the study. Subjects were given information about the study and asked to participate. All signed a consent form before participating. There was no attempt at selecting participants on any basis other than that they worked for the respective organisations and were willing to participate after learning about the study. 
Table 1 Demographic characteristics of sample and details of uses of social drugs

\begin{tabular}{ll}
\hline Characteristics & $\%$ \\
\hline Age (y): & \\
$\quad<30$ & 35.9 \\
$30-49$ & 59.0 \\
$>50$ & 5.1 \\
Marital status: & 76.9 \\
$\quad$ Married or cohabiting & 23.1 \\
Single & \\
Formal education: & 60.5 \\
$\quad \leqslant 10$ y & 15.8 \\
11-12 y & 13.2 \\
Technical college & 10.5 \\
University & 41.0 \\
Smoking history: & 25.6 \\
$\quad$ Non-smokers & 33.3 \\
Ex-smokers & $21.46(7.59)$ \\
Current smokers & \\
Smokes/day (mean (SD)) & 92.3 \\
Caffeinated beverages: & $4.29(2.01)$ \\
Consuming & \\
Drinks/day (mean (SD)) & 10.3 \\
Frequency of alcohol use: & 41.0 \\
Rarely & 43.6 \\
Weekly & 5.1 \\
2-3 Times weekly & 53.8 \\
Daily & 46.2 \\
Drinks/occasion: & \\
$\quad \leqslant 3$ & \\
₹4 & \\
\hline
\end{tabular}

MEASURES

Several performance tests were used, as described below. All tests were well established and had been used in many previously reported studies. They were chosen on the basis that they had shown sensitivity to the effects of fatigue. All tests were administered on a computer screen with a standard mouse and a keypad.

Mackworth clock

A passive vigilance test involving very low cognitive demand.

Simple reaction time

A simple response speed test.

Tracking

A low level, hand-eye coordination task with simple cognitive demands.

Dual task

A divided attention task combining the simple reaction time and tracking tests

Symbol digit test

A perceptual coding test

Spatial memory search

Memory test for a sequence of targets, with moderate complex cognitive demands and low level hand-eye coordination.

Memory and search test

A memory test with moderately complex cognitive demands and low level hand-eye coordination.
Grammatical reasoning

A logical reasoning test involving complex cognitive demands and low level hand-eye coordination.

At the beginning and end of each test session, subjects were asked to estimate their own fatigue by moving a cursor along a line on the screen corresponding to their current state of tiredness. A demographic questionnaire covering age, education level, health, recent work, and recent sleep history was administered to all subjects before the first test session. This included the Epworth sleepiness scale ${ }^{10} 11$ and three global questions on problems getting to sleep, staying asleep, and staying awake. Risk of sleep apnoea was defined as the cooccurrence of loud snoring, excessive nocturnal movement, cessation of breathing, difficulty maintaining sleep, and difficulty staying awake. ${ }^{12}$ None of the subjects showed evidence of sleep apnoea based on their questionnaire results. Before every test day, subjects were also asked about their sleep during the previous night and their food and drug intake since waking.

\section{PROCEDURES}

All testing was conducted in the laboratory. The study used a cross over randomised control design (table 2). This meant that all subjects participated in both alcohol consumption and sleep deprivation and the order of testing was counterbalanced so that half did the alcohol consumption first and the other half the sleep deprivation first. Subjects were allocated alternately to each order of testing as they entered the study. To reduce carry over effects from one condition to the other, subjects were allowed a long break in the afternoon after testing and had an overnight rest at a local motel between tests.

Subjects were tested in groups of two to six. On the afternoon before testing began, subjects spent about 4 hours doing three practice sessions for all tests. They were then sent to a local motel for an overnight rest. On the next morning testing started about 2 hours after waking, at about 0800 , for either the sleep deprivation or alcohol consumption. This was alternated for each group of subjects. The alcohol consumption involved baseline performance testing as soon as subjects arrived in the laboratory, followed by doses of alcohol at hourly intervals, with performance tests 30 minutes after each dose. Alcohol was given in four consecutive doses designed to achieve BACs of $0.025,0.05,0.075,0.1 \%$. Doses were adjusted according to percentage body fat, weight, sex, and age. ${ }^{13}$ Alcohol was given in the form of the subject's preferred variety of spirits with their preferred mixer. Alcohol measures

Table 2 Overview of the study design showing alcohol followed by the sleep deprivation

\begin{tabular}{|c|c|c|c|c|c|c|c|c|c|c|c|c|c|c|c|}
\hline Time & 0800 & 0930 & 1030 & 1130 & 1230 & 1500 & 1700 & 1900 & 2100 & 2300 & 0100 & 0300 & 0500 & 0700 & 0900 \\
\hline Day 1: practice from 1400 & & & & & & $\mathrm{~T}$ & $\mathrm{~T}$ & & & & & & & & \\
\hline Day 2: alcohol & $\mathrm{T}$ & $T^{\star}$ & $\mathrm{T}^{\star}$ & $\mathrm{T}^{\star}$ & $\mathrm{T}$ & & & & & & & & & & \\
\hline Day 3: sleep deprivation condition & $\mathrm{T}$ & $\mathrm{T}^{\star}$ & $\mathrm{T}^{\star}$ & $\mathrm{T}^{\star}$ & $\mathrm{T}$ & $\mathrm{T}$ & $\mathrm{T}$ & $\mathrm{T}$ & $\mathrm{T}$ & $\mathrm{T}$ & $\mathrm{T}$ & $\mathrm{T}$ & $\mathrm{T}$ & $\mathrm{T}$ & $\mathrm{T}$ \\
\hline
\end{tabular}

$\mathrm{T}=$ performance test; $\mathrm{T}^{\star}=$ short version of test.

For half of the subjects the order of alcohol and sleep deprivation was reversed. 
(blood-breath equivalents) were taken with a breathlyser (Drager Alcotest 7110) immediately before and after each test session and then hourly until the subjects' alcohol concentrations were below $0.05 \%$. The subjects were then allowed to leave the test centre.

The sleep deprivation involved the same sequence of testing as for the alcohol consumption, with tests every hour from baseline to the 5 th hour, as in the alcohol consumption, then every 2 nd hour for the next 20 hours. The last test occurred 28 hours after waking. There were five performance test sessions in all for the alcohol consumption and 15 for the sleep deprivation. The order of tests was randomised within each test session. Most tests were used in all sessions: however, three tests (grammatical reasoning, spatial memory search, and memory and search task) were dropped from the second, third, and fourth test sessions to allow time for alcohol to be drunk and absorbed. This procedure was also used for the sleep deprivation so that direct comparisons could be made between the conditions.

\section{STATISTICAL ANALYSIS}

For statistical analysis, for each subject the BACs recorded at the start and end of each of the five test sessions were averaged to produce a single value for each test session. These average BACs and the associated performance test measures were plotted separately against test session for each subject. Due to individual differences in absorption of alcohol, the observed BACs were not always at the anticipated level at each test session. This meant that the time at which the exact BACs of $0.025 \%, 0.05 \%$, $0.075 \%$, and $0.1 \%$ were reached had to be interpolated from the graph for each subject. These times were then identified on the graph of performance test measures against test session and the corresponding test scores could then be interpolated for each subject. By this method it was possible to estimate the performance test score corresponding to each concentration of alcohol for each subject. These concentrations were then averaged across subjects to show change in performance with each alcohol dose.

Performances at the BACs of $0.05 \%$ and $0.1 \%$ were then compared with performance across sleep deprivation test sessions 8-13 (at times 1900-0500) for each subject. This time window was chosen for the sleep deprivation condition before the data were collected because this period was most likely to produce effects of fatigue as it covered the longest periods of sleep deprivation and covered the period of the major circadian trough. This decision was reinforced when the sleep deprivation and performance relation was plotted after data collection, as this period also showed the clearest linear trend across test sessions for all measures. For this analysis, time was treated as a continuously increasing quantity across midnight, for example, 20 hours of sleep deprivation occurred at 0200 , as the waking time had been about 0600 . Over this time window, the sessions between which performance under the sleep deprivation first became worse than the
Table 3 Amount and quality of sleep the night before alcohol and sleep deprivation

\begin{tabular}{lcc}
\hline & $\begin{array}{l}\text { Before alcohol } \\
\text { Mean (SD) }\end{array}$ & $\begin{array}{l}\text { Before sleep } \\
\text { deprivation } \\
\text { Mean (SD) }\end{array}$ \\
\hline $\begin{array}{l}\text { Amount of sleep } \\
\text { Rated quality of sleep }\end{array}$ & $7.5(2.4)$ & $7.2(1.0)$ \\
Ratings of freshness at waking & $72.7(25.0)$ & $58.7(24.9)$ \\
\hline
\end{tabular}

performance found at BACs of $0.05 \%$ and $0.1 \%$ were noted for each subject. With interpolation, the time since waking associated with performance equivalent to that at the two alcohol concentrations were then identified for each subject. The scores for time since waking were then averaged across subjects for each performance measure.

Not all subjects contributed to the time since waking scores for each measure as not all subjects showed a deterioration in performance over this time window for all performance tests. Only data from subjects who showed a change from better than the BACs of $0.05 \%$ and $0.1 \%$ to worse than these criterion concentrations over the 1900 to 0500 window were included in the averages for each test. The number of subjects contributing to each hour of wakefulness equivalent to the BACs therefore reflects the percentage of subjects who showed significant deterioration in performance over the selected time window.

\section{Results}

Subjects were reasonably well rested after a mean of 7.54 and 7.16 hours overnight sleep immediately before each test condition, for alcohol comsumption and sleep deprivation respectively (table 3). Although sleep quality was rated as significantly lower before sleep deprivation, the amount of sleep and ratings of feeling fresh after waking did not differ between the conditions, indicating that subjects were not partially sleep deprived before either test condition.

As expected, increasing concentrations of alcohol produced significant reductions in performance for most tests and measures. Table 4 shows the results of the estimated change in performance due to varying amounts of alcohol compared with baseline, no alcohol. The results show that the extent of loss of function varies between tests although there were consistent effects within different types of measures. At a BAC of $0.05 \%$ for example, response speed decreased by around $8 \%-15 \%$ for reaction time, dual task, Mackworth vigilance, and symbol digit tests corresponding to a slowing of around 45, 66, 136, and $182 \mathrm{~ms}$ respectively. Hand-eye coordination measures showed a similar overall decrement of around $10 \%$ at this BAC. Measures of overall test accuracy also showed significant decrements due to alcohol, especially the number of missed signals in the reaction time test, which increased by $200 \%$, and the number of false alarm responses in the Mackworth test, which were more than $50 \%$ higher at a BAC of $0.05 \%$. The number of correct responses in the Mackworth test and length of the recalled series in the spatial memory task also both 
Table 4 Interpolated performance estimates at baseline and with blood alcohol (BAC) at certain concentrations

\begin{tabular}{|c|c|c|c|c|}
\hline \multirow[b]{2}{*}{ Test } & \multirow[b]{2}{*}{ Measure } & \multirow[b]{2}{*}{ Baseline 0.00} & \multicolumn{2}{|l|}{$B A C(\%)$} \\
\hline & & & 0.05 & 0.1 \\
\hline \multirow[t]{2}{*}{ Reaction time } & Speed (ms) & 489 & 534 & 566 \\
\hline & Accuracy (misses) & 0.36 & 1.17 & 2.81 \\
\hline \multirow[t]{2}{*}{ Dual task } & Speed (ms) & 662 & 725 & 792 \\
\hline & Hand-eye coordination difficulty level & 50.59 & 45.43 & 23.69 \\
\hline Tracking & Hand-eye coordination difficulty level & 47.76 & 44.35 & 23.39 \\
\hline \multirow[t]{3}{*}{ Mackworth } & Speed $(\mathrm{ms})$ & 958 & 1094 & 1361 \\
\hline & Accuracy (targets detected (n)) & 12.64 & 10.91 & 7.76 \\
\hline & Accuracy (false alarms) & 1.05 & 1.63 & 4.48 \\
\hline \multirow[t]{3}{*}{ Symbol digit } & Speed $(\mathrm{ms})$ & 2233 & 2415 & 2656 \\
\hline & Speed (targets inspected $(\mathrm{n}))$ & 40.11 & 37.32 & 32.74 \\
\hline & Accuracy $($ correct $(\%))$ & 99.00 & 97.83 & 94.52 \\
\hline \multirow[t]{2}{*}{ Grammatical reasoning* } & Speed (ms) & 4286 & 4135 & 3945 \\
\hline & Accuracy (correct $(\mathrm{n}))$ & 23.19 & 21.89 & 20.05 \\
\hline \multirow[t]{4}{*}{ Memory and search ${ }^{\star}$} & Speed (ms) -2 targets & 12222 & 12399 & 12500 \\
\hline & Speed $(\mathrm{ms})-6$ targets & 20853 & 20302 & 19555 \\
\hline & Accuracy (correct $(\mathrm{n}))-2$ targets & 5.59 & 5.31 & 5.01 \\
\hline & Accuracy (correct $(n))-6$ targets & 5.05 & 4.66 & 4.21 \\
\hline Spatial memory* & Length of recalled series & 5.34 & 4.65 & 3.73 \\
\hline Tiredness & Rating & 17.84 & 31.63 & 44.83 \\
\hline
\end{tabular}

*Performance estimates based on only the first and last test occasion.

decreased by about $13 \%$ at a BAC of $0.05 \%$. Subjective ratings of tiredness also showed a significant linear decrement of $77 \%$ by a BAC of $0.05 \%$. Two tests, grammatical reasoning and memory and search tests showed very little decrease in performance at a BAC of $0.05 \%$.

At a BAC of $0.1 \%$ performance was poorer for all measures for all tests and some measures showed more than twice the decrement at a BAC of $0.05 \%$. The biggest changes were seen for the accuracy measures, number of misses in the reaction time test, which was nearly seven times poorer at a BAC of $0.1 \%$ than at baseline, and the number of false alarms for the Mackworth vigilance test, which increased to three times the level at baseline. Hand-eye coordination in both tracking and dual tasks also showed a much larger decrement than other tests, with a $50 \%$ deterioration at this BAC. Response speed for the Mackworth test also deteriorated more than might be expected and showed $42 \%$ slowing compared with baseline. By comparison, the other measuresresponse time for the simple reaction time, dual task and symbol digit tests, the spatial memory test, and subjective ratings of fatigue-all showed around twice as much deterioration at a BAC of $0.1 \%$ than $0.05 \%$. Similarly, the higher cognitive tests, logical reasoning, and memory and search also showed around twice the level of deterioration at this BAC, but the level of deterioration was still quite small (around 10\%), even at this higher level of alcohol.

These results show that alcohol does not exert universal effects on all functions and the pattern of effects also differs between them.

Sleep deprivation also produced decrements in both performance and self rated alertness. As shown in table 5, sleep deprivation showed effects on a similar range of tests as did alcohol. At the beginning of the analysed time window (1900) performance for most tests was very similar to performance during the first session of the sleep deprivation test day. Over the time window, however, performance decrements occurred with increasing sleep deprivation for both speed and accuracy measures of the reaction time, dual task, tracking, and Mackworth tests and for the length of the recalled series for the spatial memory test. For example, between around 1900 and 0500 (corresponding to

Table 5 Interpolated performance estimates as a function of time of day (hours since waking where average waking time was 0544) during the selected sleep deprivation time window

\begin{tabular}{|c|c|c|c|c|c|c|}
\hline \multirow[b]{2}{*}{ Test } & \multirow[b]{2}{*}{ Measure } & \multirow[b]{2}{*}{$\begin{array}{l}\text { First sleep test } \\
\text { session } 0800 \\
(2.27)\end{array}$} & \multirow[b]{2}{*}{$\begin{array}{l}\text { Start of analysed } \\
\text { window } 1900 \\
(13.27)\end{array}$} & \multicolumn{3}{|c|}{ Time of day (hours since waking) } \\
\hline & & & & $\begin{array}{l}1944 \\
(14.00)\end{array}$ & $\begin{array}{l}2344 \\
(18.00)\end{array}$ & $\begin{array}{l}2744 / 0344 \\
(22.00)\end{array}$ \\
\hline \multirow{2}{*}{ Reaction time } & Speed (ms) & 494 & 495 & 497 & 521 & 540 \\
\hline & Accuracy (misses) & 0.69 & 1.08 & 0.98 & 1.67 & 3.10 \\
\hline \multirow[t]{2}{*}{ Dual task } & Speed (ms) & 618 & 617 & 627 & 709 & 775 \\
\hline & Hand-eye coordination difficulty level & 48.84 & 48.31 & 49.11 & 46.62 & 33.37 \\
\hline Tracking & Hand-eye coordination difficulty level & 44.07 & 49.52 & 47.66 & 40.83 & 36.70 \\
\hline \multirow[t]{3}{*}{ Mackworth } & Speed $(\mathrm{ms})$ & 1020 & 964 & 1010 & 1225 & 1511 \\
\hline & Accuracy (targets detected (n)) & 12.77 & 12.00 & 11.89 & 9.86 & 7.04 \\
\hline & Accuracy (false alarms) & 2.15 & 1.28 & 1.48 & 2.85 & 4.24 \\
\hline \multirow[t]{3}{*}{ Symbol digit } & Speed (ms) & 2289 & 2245 & 2282 & 2430 & 2577 \\
\hline & Speed (targets inspected $(\mathrm{n})$ ) & 38.49 & 40.05 & 39.30 & 36.90 & 34.30 \\
\hline & Accuracy (correct $(\%))$ & 98.05 & 98.32 & 98.29 & 98.37 & 97.41 \\
\hline \multirow[t]{2}{*}{ Grammatical reasoning } & Speed (ms) & 4413 & 4054 & 4128 & 4255 & 4182 \\
\hline & Accuracy (correct $(\mathrm{n}))$ & 21.62 & 23.59 & 23.13 & 22.76 & 22.46 \\
\hline \multirow[t]{4}{*}{ Memory and search } & Speed $(\mathrm{ms})-2$ targets & 11988 & 11336 & 11620 & 12439 & 12581 \\
\hline & Speed $(\mathrm{ms})-6$ targets & 22423 & 20729 & 20787 & 21460 & 21101 \\
\hline & Accuracy (correct $(n))-2$ targets & 5.54 & 5.65 & 5.57 & 5.37 & 5.35 \\
\hline & Accuracy (correct $(n))-6$ targets & 5.08 & 5.16 & 5.14 & 5.12 & 4.80 \\
\hline Spatial memory & Length of recalled series & 5.25 & 5.15 & 5.14 & 4.87 & 4.27 \\
\hline Tiredness & Rating & 19.87 & 38.74 & 40.52 & 58.62 & 75.47 \\
\hline
\end{tabular}

Performance during the first test session of the sleep deprivation is included for comparison with the start of the selected window. 
Table 6 Equating the effects of sleep deprivation and alcohol consumption

\begin{tabular}{|c|c|c|c|c|c|c|}
\hline \multirow[b]{3}{*}{ Test and measure } & \multicolumn{6}{|c|}{ Hours (decimal) of wakefulness equivalent to $B A C$ concentrations } \\
\hline & \multicolumn{3}{|c|}{$B A C 0.05 \%$} & \multicolumn{3}{|c|}{$B A C 0.1 \%$} \\
\hline & Mean & $95 \% C I$ & $\%^{\star}$ & Mean & $95 \% C I$ & $\%$ * \\
\hline \multicolumn{7}{|l|}{ Reaction time task: } \\
\hline Speed $(\mathrm{ms})$ & 18.04 & 17.12 to 18.96 & 76 & 18.71 & 17.56 to 19.86 & 64 \\
\hline Accuracy (misses) & 17.31 & 16.51 to 18.11 & 42 & 17.74 & 16.51 to 18.97 & 45 \\
\hline \multicolumn{7}{|l|}{ Dual task: } \\
\hline Speed (ms) & 17.73 & 16.75 to 18.71 & 84 & 19.65 & 18.58 to 20.77 & 67 \\
\hline Hand-eye coordination (level of difficulty) & 18.43 & 17.41 to 19.45 & 79 & 19.42 & 18.40 to 20.44 & 58 \\
\hline \multicolumn{7}{|l|}{ Tracking task: } \\
\hline Hand-eye coordination (level of difficulty) & 18.25 & 17.37 to 19.13 & 74 & 19.01 & 18.91 to 19.97 & 61 \\
\hline \multicolumn{7}{|l|}{ Mackworth clock vigilance: } \\
\hline Speed $(\mathrm{ms})$ & 17.08 & 16.20 to 17.96 & 82 & 18.10 & 16.85 to 19.35 & 58 \\
\hline Accuracy (misses) & 17.64 & 16.72 to 18.56 & 68 & 18.80 & 17.93 to 19.67 & 76 \\
\hline \multicolumn{7}{|l|}{ Symbol digit task: } \\
\hline Speed (ms) & 18.55 & 17.43 to 19.67 & 50 & 18.91 & 17.92 to 19.90 & 48 \\
\hline Speed (symbols inspected (n)) & 18.52 & 17.46 to 19.58 & 57 & 18.64 & 17.65 to 19.63 & 79 \\
\hline Accuracy (correct $(\%))$ & 16.91 & 15.72 to 18.10 & 41 & 18.39 & 17.01 to 19.77 & 42 \\
\hline \multicolumn{7}{|l|}{ Spatial memory task: } \\
\hline Accuracy (length of recalled sequence) & 18.05 & 17.09 to 19.01 & 86 & 17.88 & 16.92 to 18.84 & 64 \\
\hline
\end{tabular}

${ }^{\star}$ Numerator=number of subjects contributing data; denominator=number of subjects whose range of BAC incorporated $0.05 \%$ $(n=37$ or 38$)$ or $0.1 \%(n=33)$.

Amount of sleep deprivation required to produce performance decrements equivalent to varying concentrations of blood alcohol (BAC), and the time of day at which the equivalence occurred in this study.

about 13-23 h sleep deprivation), reaction speed decreased by $57 \%$ for the Mackworth test, $9 \%$ for reaction time, $27 \%$ for dual task and $15 \%$ for symbol digit tests. Hand-eye coordination decreased by between $31 \%$ for the tracking component of the dual task and $26 \%$ for the tracking task alone. Accuracy also decreased markedly with sleep deprivation. The number of missed signals increased by more than $40 \%$ for the Mackworth test, by $187 \%$ for the reaction time test, and the number of false alarms increased by $200 \%$ for the Mackworth test. The symbol digit test only showed decrements for the speed measures but not the accuracy measure. The grammatical reasoning and memory and search tasks showed only relatively small decreases of around 5\%-10\% with increasing sleep loss for any measures.

The levels of sleep deprivation estimated to produce decrements in performance equivalent to varying concentrations of alcohol are shown for each performance measure in table 6 . The results indicate that on average, $0.05 \%$ equivalence occurred after being awake for around 16.91 to 18.55 hours, placing the time of the effect in this study to between 2238 and 0017 . At a BAC of $0.1 \%$, equivalence occurred after between 17.74 and 19.65 hours of wakefulness which falls in the late evening to early hours of the morning, corresponding in this study to between 2328 and 0123 . Measures within and between tests were affected at very similar levels of sleep deprivation. The performance test that seemed to be affected first was the passive vigilance test, the Mackworth clock test, where equivalence to a BAC of $0.05 \%$ occurred after just over 17 hours of wakefulness for all measures. The accuracy measure of the symbol digit test reached levels equivalent to $0.05 \%$ alcohol earlier than any other measure for any test, but equivalence occurred considerably later for the other symbol digit test measures. The likelihood of missing targets in the reaction time test was also affected by sleep deprivation slightly earlier than other tests equivalent to a BAC of $0.05 \%$ as it also occurred at just over 17 hours of wakefulness. The two tests that showed little change with increasing sleep loss, grammatical reasoning and memory and search tasks, were not included in this analysis as alcohol equivalences are likely to be misleading.

Table 6 shows that the percentage of subjects showing poorer performance than a BAC of $0.05 \%$ and $0.1 \%$ across the session $8-13$ window varied considerably between tests. More than three quarters of subjects showed deterioration in performance to become poorer than the BAC of $0.05 \%$ for speed measures in the simple reaction time, dual task, and Mackworth clock vigilance tests, and in the accuracy of the spatial memory search test. By contrast, for the accuracy measures of the simple reaction time and symbol digit tasks only around $40 \%$ of subjects showed performance decrements sufficient to be at or poorer than the BAC of $0.05 \%$. As might be expected, for most tests, a smaller percentage of subjects showed performance levels equivalent to a BAC of $0.1 \%$. Nevertheless for most tests, more than half of the subjects showed deterioration in performance equivalent to a $\mathrm{BAC}$ of $0.1 \%$. Fewer subjects reached a BAC of $0.1 \%$ for the accuracy measures of reaction time and symbol digit tests, as was found for $0.05 \%$ equivalence. For a few measures, more subjects reached equivalence to a $\mathrm{BAC}$ of $0.1 \%$ than $0.05 \%$, notably, accuracy on the Mackworth test, and the number of symbols inspected in the symbol digit test. This finding is most likely because these measures had a performance ceiling and many subjects remained at the ceiling, even at a BAC of $0.05 \%$, and only showed a performance decrement between the BACs of $0.05 \%$ and $0.1 \%$.

\section{Discussion}

This study shows that commonly experienced levels of sleep deprivation depressed performance to a level equivalent to that produced by alcohol intoxication of at least a BAC of $0.05 \%$. At the end of periods of waking of 17-19 hours, performance levels were low enough to be 
accepted in many countries as incompatible with safe driving. The earliest effects were seen for the Mackworth clock test and the latest for the dual task, although there was relatively little variation across tests. Longer periods of sleep deprivation were equivalent to higher alcohol doses for all tests except the grammatical reasoning and memory and search tasks.

Equivalence with the BAC of $0.05 \%$ was also very similar within tests. Both parts of the dual task, either when tested alone, or in combination, showed equivalence at between 17 and 19 hours of sleep loss corresponding in this study to between 2240 and 0050 . For all measures of the Mackworth clock test, equivalence occurred after around 17 hours of sleep loss and for the symbol digit test after about 17-19 hours without sleep.

These results show that impairments in performance which have been judged as the legal limit for driving safely may start to occur as early as 17 hours after waking and around 18 hours on average after waking. These results confirm earlier work on a single task. ${ }^{9}$ It is important that these periods of wakefulness also correspond to the normal waking day for most people. In the community a 16-17 hour period of wakefulness would be regarded as normal, with bedtime typically occurring in the mid to late evening depending on the time of rising. It could be argued, therefore, that this common pattern of waking and sleeping plays a major part in ensuring safety. If the period of wakefulness is extended beyond the usual 16-17 hours, performance is likely to be impaired sufficiently to represent a considerably greater risk of injury. Driving home after a long work day, for example, may put you at increased risk of an accident. Drivers who have been awake for more than 17-18 hours are likely to be significantly slower at reacting and will be increasingly likely to miss information as the period of sleep loss increases even further.

This study looked at effects of sleep deprivation only under day worker conditions where subjects were rested after a reasonable number of hours sleep the night before. Although most people follow this sleep-waking regime, work schedules and lifestyle demands increasingly require people to extend their waking period for longer than 18 hours, shortening their sleeping period as a consequence, and to do so repeatedly over days, weeks, or even months. The effects of such chronic partial sleep deprivation have not been considered by this study although these findings and a recent review of the literature ${ }^{14}$ suggest that partial sleep deprivation may present very serious risk for safe performance.

Although this study has not directly considered the role of circadian effects, it is known that they interact with deficits in performance from continuous or partial sleep deprivation. ${ }^{15}$ This study was designed to only look at the effects of sleep loss over a night without sleep after a day awake as this is the form that sleep loss often takes. This meant, however that the period of maximum sleep loss coincided with the time that circadian influences should have been greatest. As a result, performance deficits may have been higher for measures that were vulnerable to circadian influences so enhancing the apparent effects of sleep loss. Further research is needed to clarify the relative effects of sleep deprivation and circadian influences and to measure them against the alcohol comsumption benchmark. It is notable, however, that the deficits from sleep deprivation found here equivalent to a BAC of $0.05 \%$, occurred between 2200 and 0000 , which is well ahead of the time at which the circadian trough occurs. ${ }^{15} 16$ This suggests that sleep deprivation and not circadian influences causes serious concern about decrements in performance, although our results show the additional deterioration in performance due to circadian effects.

The overall implications of the results of this study are clear. They show that the effects on performance of moderate periods of being awake cannot be discounted. Sleep is needed after the end of a day if adverse effects on performance are to be avoided. Most importantly, this study has allowed interpretation of these effects on performance in terms of an accepted standard for safety. With a legal limit for alcohol use when driving as a standard, the results show that after 17-19 hours of wakefulness, subjects' performance on many tests had dropped to that found at the legal limits for safe driving. Many people remain awake for periods of 16 hours or more for reasons of work, family, or social life. These results suggest that after this duration of wakefulness fatigue reaches a level that can compromise safe performance.

The results also imply that many countries which set allowable BACs at the point that compromises safe performance should consider developing similar standards for fatigue to ensure that people who have had 18 hours or longer without sleep are kept from at risk behaviours such as driving, piloting aircraft, or operating machinery.

We thank the subjects for their cooperation throughout the study. We also thank Rena Friswell and Samantha Finlay-Brown for their research assistance, Associate Professor Richard Mattick for assistance with alcoho staff of the National Drug and Alcohol Research Centre for permitting us to use their facilities. The project was funded by the Federal Office of Road Safety.

1 Horne J, Reyner LA. Sleep related vehicle accidents. BMF 1995;310:565-7.

2 Sweatman PF, Ogden KJ, Haworth $\mathrm{N}$, et al. NSW heavy vehicle crash study. Canberra: Federal Office of Road Safety, 1990.

3 Leger D. The cost of sleep-related accidents: a report for the National Commission on Sleep Disorders Research. Sleep 1994;17:84-93.

4 Mitler M, Casakadon MA, Czeisler CA, et al. Catastrophes, sleep, and public policy: consensus report. Sleep 1988;11: $100-9$

5 Report on the National Commission on Sleep Disorders Research. Wake up America: a national sleep alert. Washington, DC: US Department of Health and Human Services, January 1993.

6 Dement W C. The perils of drowsy driving. $N$ Engl f Med 1997;333:783-4.

7 Committee on the benefits and costs of alternative federal blood alcohol concentration standards for commercial vehicle operators. Zero alcohol and other options: Limits for ruck and bus drivers. Washington, DC: Transportation Research Board, 1987.

8 Howat P, Sleet D, Smith I. Alcohol and driving: Is the $0.05 \%$ blood alcohol concentration limit justified? Drug and Alcohol Review 1991;10:151-66.

9 Dawson A, Reid K. Fatigue, alcohol, and performance Dawson A, Reid K. Fatigue, alco
impairment. Nature 1997;388:235. 
10 Johns MW. A new method for measuring daytime sleepiness: The Epworth sleepiness scale. Sleep 1991;14: 5leepiness:

11 Johns, MW. Reliability and factor analysis of the Epworth sleepiness scale. Sleep;15:376-81.

12 Haraldsson P-O, Carenfeld C, Tingvall C. Sleep apnea syndrome symptoms and automobile driving in a general population. F Clin Epidemiol 1992;45:821-5.

13 Durnin JVGA, Womersley J. Body fat assessed from total body density and its skinfold thickness: measurements on 481 men and women aged from 16 to 72 years. $\mathrm{Br} \mathcal{F}$ Nutr 1974;32:77-97.
14 Pilcher JJ, Huffcutt AI. Effects of sleep deprivation on performance: a meta-analysis. Sleep 1996;19:318-26.

15 Moore-Ede M, Campbell S, Baker T. Falling asleep at the wheel: research priorities to improve driver alertness and highway safety. Federal Highway Administration Symposium on Truck and Bus Driver Fatigue, 29-30 November 1988. Washington, DC: Federal Highway Administration, 1998.

16 Kryger MH, Roth T, Carskadon M. Circadian rhythms in humans: an overview. In: Kryger $\mathrm{MH}$, Roth $\mathrm{T}$, Dement WC, eds. Principles and practice of sleep medicine, 2nd ed. Philadelphia: WB Saunders, 1994:301-8.

\section{Vancouver style}

All manuscripts submitted to Occup Environ Med should conform to the uniform requirements for manuscripts submitted to biomedical journals (known as the Vancouver style.)

Occup Environ Med, together with many other international biomedical journals, has agreed to accept articles prepared in accordance with the Vancouver style. The style (described in full in the $\mathcal{F} A M A[1]$ ) is intended to standardise requirements for authors, and is the same as in this issue.

References should be numbered consecutively in the order in which they are first mentioned in the text by Arabic numerals on the line in square brackets on each occasion the reference is cited (Manson[1] confirmed other reports[2][3][4][5]). In future references to papers submitted to Occup Environ Med should include: the names of all authors if there are three or less or, if there are more, the first three followed by et al; the title of journal articles or book chapters; the titles of journals abbreviated according to the style of Index Medicus; and the first and final page numbers of the article or chapter. Titles not in Index Medicus should be given in full.

Examples of common forms of references are:

1 International Committee of Medical Journal Editors. Uniform requirements for manuscripts submitted to biomed journals. $7 A M A$ 1993;269:2282-6.

2 Soter NA, Wasserman SI, Austen KF. Cold urticaria: release into the circulation of histmaine and eosinophil chemotactic factor of anaphylaxis during cold challenge. N Engl F Med 1976;294:687-90.

3 Weinstein L, Swartz MN. Pathogenic properties of invading micro-organisms. In: Sodeman WA Jr, Sodeman WA, eds. Pathologic physiology, mechanisms of disease. Philadelphia: W B Saunders, 1974:457-72. 